
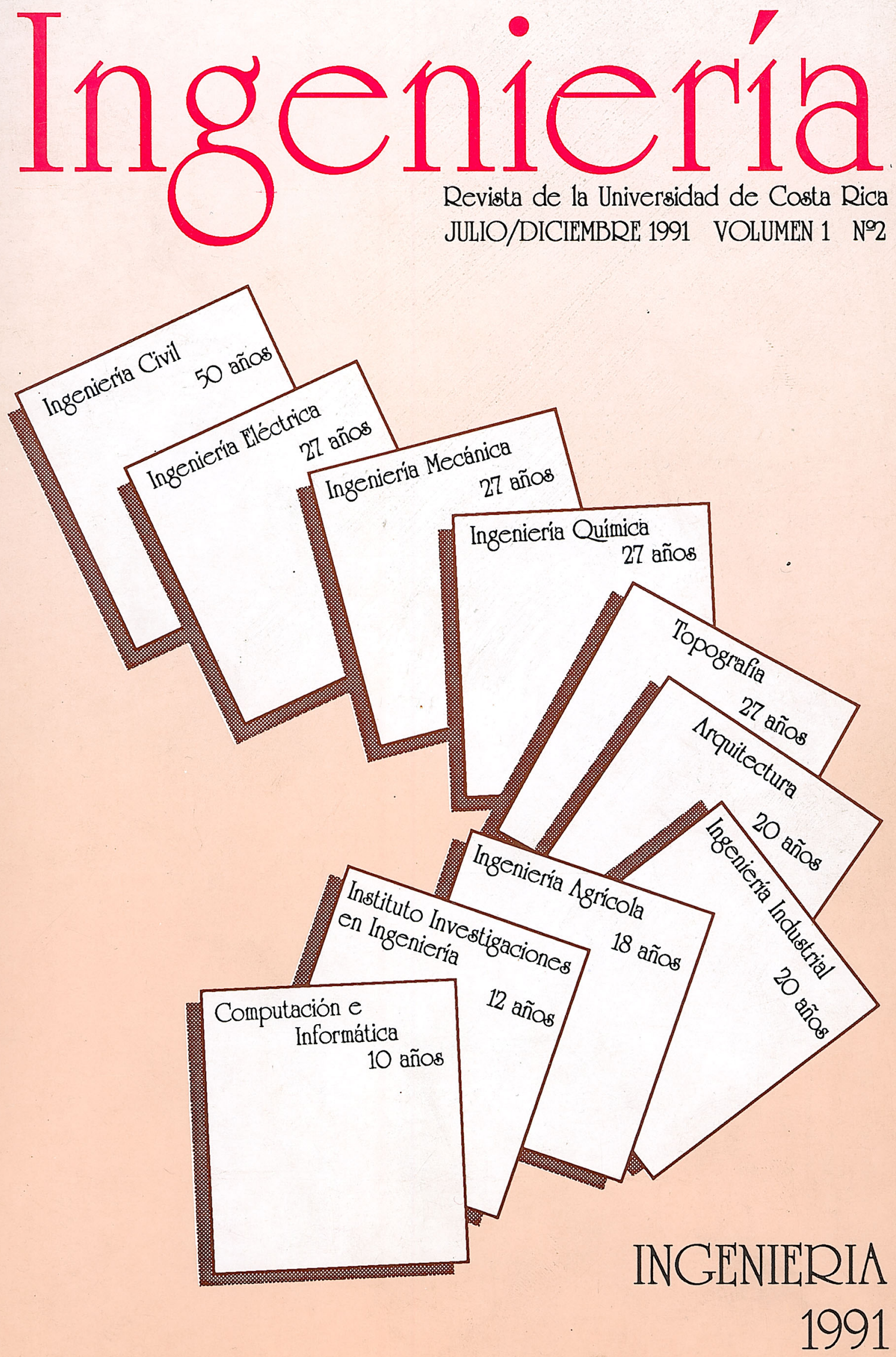


\section{DESILIZACION DE LA PULPA BRUTA OBTENIDA EN EL TRATAMIENTO DE LAS BAUXITAS DEL VALLE DEL GENERAL POR EL PROCESO ALCALINO BAYER. A PRESION ATMOSFERICA}

\section{Resumen}

Se ha llevado a cabo una investigación sobre el efecto de la temperatura, tiempo de agitación y la variación de la cantidad de $\mathrm{CaO}$ utilizada, en el proceso de desilización de la pulpa bruta obtenida en el tratamiento de las bauxitas a presión atmosférica por el proceso alcalino Bayer. Se determinó que el aumento de las variables antes indicadas favorecen el proceso de desilización.

\section{Summary}

A research has been done on the effect of temperature, mixing time, and the quantity of $\mathrm{CaO}$ on the desilication process of the gross pulp obtained from bauxite treated by the Bayer alcaline process at atmospheric pressure. It was determined that an increase on the value of the mentioned variables is favourable to the desilication process.

\section{CONSIDERACIONES TEORICAS}

En la tecnología del tratamiento de las bauxitas por el proceso Bayer, la presencia del sílice, en concentraciones pequeñas en la materia prima sujeta a las primeras fases tecnológicas, no constituye un impedimento. Cuando el contenido de sílice sobrepasa un determinado valor (sobre 6\%) es necesaria la ejecución de una operación de desilización.

El silice amorfo y el caolín se disuelven incluso en el momento del molido de la bauxita (en presencia de soluciones alcalinas), mientras que la disolución del cuarzo tiene lugar a una presión y temperatura relativamente altas /1/y por esta causa este proceso se lleva a cabo en la fase de solubilización en autoclave. En numerosas investigaciones experimentales se ha establecido que en el proceso de solubilización de las bauxitas y los compuestos de sílicio (tanto las formas amorfas, como también las cristalinas interaccionan con las soluciones alcalinas o de aluminatos sien-

* Escuela de Ingeniería Química Facultad de Ingeniería Universidad de Costa Rica. do transformadas en alumo-silicatos de sodio hidratados $/ 2,3 /$ poco solubles.

Las siguientes reacciones /1,5/muestran la formación de alumo-silicatos de sodio hidratados debido a la formacion, en las soluciones de aluminato sobresaturados en $\mathrm{SiO}_{2}$, (equilibrio metaestable $/ 4,5,6 /$ de un complejo $\left(\mathrm{mNa}{ }_{2} \mathrm{O} . \mathrm{Al}_{2} \mathrm{O}_{3} .2 \mathrm{SiO}_{2}\right)$ el cual por hidrólisis se transforma en el hidroalumo-silicato de sodio.

$$
\mathrm{HAlO}_{2}+\mathrm{NaOH}=\mathrm{NaAlO}_{2}+\mathrm{H}_{2} \mathrm{O}
$$

$$
\mathrm{H}_{3} \mathrm{AlO}_{3}+\mathrm{NaOH}=\mathrm{NaAlO}_{2}+2 \mathrm{H}_{2} \mathrm{O}
$$

(2)

$\mathrm{SiO}_{2}+2 \mathrm{NaOH}=\mathrm{Na}_{2} \mathrm{SiO}_{3}+\mathrm{H}_{2} \mathrm{O}$

(3)

$$
\begin{array}{r}
2 \mathrm{NaAlO}_{2}+2 \mathrm{Na}_{2} \mathrm{SiO}_{3}+\mathrm{nNaOH}+\mathrm{aq} \\
\begin{array}{r}
\mathrm{mNa}_{2} \mathrm{O}_{2} \mathrm{Al}_{2} \mathrm{O}_{3} \cdot 2 \mathrm{SiO}_{2} \\
\text { complejo aluminosilísico }
\end{array}
\end{array}
$$


(4)

$$
+(\mathrm{m}+6-2 \mathrm{~m}) \mathrm{NaOH}+\mathrm{aq}
$$

$\mathrm{mNa}_{2} \mathrm{O}-\mathrm{Al}_{2} \mathrm{O}_{3}-2 \mathrm{SiO}_{2}+\mathrm{aq}=$

(5)

$$
\begin{aligned}
& \text { hidroalumo-silicato de sodio } \\
& \text { (permutito) } \\
& +2(\mathrm{~m}-1) \mathrm{NaOH}+\mathrm{aq}
\end{aligned}
$$

La interacción de los compuestos de silicio con las soluciones alcalinas de aluminatos se desarrolla, a temperaturas bajas $\left(95-100{ }^{\circ} \mathrm{C}\right)$ y en un intervalo grande de concentraciones. La velocidad de disolución depende de una serie de factores, tales como: temperatura, concentración de $\mathrm{Na}_{2} \mathrm{O}$ y $\mathrm{Al}_{2} \mathrm{O}_{3}$ y en especial de la naturaleza y forma cristalina de los minerales presentes en la bauxita.

El contenido de sílice de las soluciones de aluminato debe investigarse permanentemente debido al hecho de que la alúmina obtenida al final de la fase de calcinación, para poder ser utilizada en el proceso de electrólisis, no debe contener más de $0,035 \% \mathrm{SiO}_{2}$.

Para establecer las posibilidades de minimización del efecto dañino del sílice se han efectuado numerosos estudios con respecto al límite de solubilización del silice, a la velocidad de desilización, a la naturaleza física y química de los productos de desilización, etc.

La diversidad de los procesos de desilización $77 /$ deriva del interés de reducir la solubilidad real del $\mathrm{SiO}_{2}$, con el crecimiento concomitante de la velocidad de su deposición (adiciones de $\mathrm{CaO}$, $\mathrm{CaCO}_{3}, \mathrm{Na}_{2} \mathrm{CO}_{3}, \mathrm{NaCl}$, etc.) o solamente de intensificar el proceso físico de deposición del $\mathrm{SiO}_{2}$ sin el cambio de las solubilidades reales (crecimiento de la velocidad de agitación, aumento de la cantidad de agente de descomposición, etc.)

Numerosas investigaciones y la práctica de la fabricación de la alúmina muestran que los agregados químicos tienen una influencia positiva sobre el grado de desilización de las soluciones de aluminatos. Entre los agregados químicos más estudiados y usados en la desilización de las soluciones de aluminatos están: 6́xido de calcio, barro rojo y gris, barro blanco y soda.

$\mathrm{El} \mathrm{CaO}$ forma alumo-silicato de calcio el cual es menos soluble que el alumo-silicato de sodio /8,9,10/. Por esta causa la separación del silice de la solución se acelera en presencia del agregado de $\mathrm{CaO}$. Se presupone, que el acelerador del óxido de calcio en la separación del silice lleva también al mejoramiento de la cristalización del alumo-silicato de sodio.

Para lograr unos grados de desilización máximos son necesarias concentraciones mayores de $10 \mathrm{~g} / \mathrm{L} \mathrm{CaO}$; esta concentración es cerca de 6 a 8 veces mayor que la cantidad estequiométrica necesaria para ligar todo el SiO bajo la forma: $\mathrm{CaO}$ - $\mathrm{Al}_{2} \mathrm{O}_{3} \cdot 1,7 \mathrm{SiO}_{2} \cdot \mathrm{nH}_{2} \mathrm{O}$.

La velocidad del proceso de desilización es directamente proporcional con la cantidad de $\mathrm{CaO}$ agregada, pero una vez con el crecimiento del contenido de $\mathrm{CaO}$; aumentan también las pérdidas de alúmina que se separa no sólo con el alumo-silicato de calcio, sino también bajo la forma de hidroaluminato de calcio $\left(3 \mathrm{CaO} \mathrm{AL}_{2} \mathrm{O}_{3}\right.$ $6 \mathrm{H}_{2} \mathrm{O}$ ). Por esta causa no es conveniente que la desilización se haga en presencia de grandes cantidades de $\mathrm{CaO}$.

En el caso de un contenido elevado de sílice en la bauxita para evitar las incrustaciones de alumo-silicatos en los tubos de los intercambiadores de calor, se efectúa una desilización de la pulpa antes de la fase de solubilización por el mantenimiento de ésta, bajo agitación, en un lapso de varias horas a una temperatura bastante cercana a $100^{\circ} \mathrm{C}$. De los datos suministrados por la literatura $/ 11$ se observa que en el período inicial de contacto del súlice con la solución de aluminato, la velocidad de solubilización del silice sobrepasa a la velocidad de formación del alumo-silicato poco soluble, así mismo en el curso de las primeras 2 a 3 horas de interacción, en la solución se establece la concentración máxima metaestable de silice. A continuación tiene lugar el proceso de desilización con la formación del hidroalumo-silicato poco soluble. El contenido de silice en la solución de aluminato en el equilibrio está en función de la solubilidad del hidroalumo-silicato. El proceso de transformación del sílice de la bauxita en hidroalumo-silicato transcurre en dos etapas: la disolución del silice en la solución de aluminato y la cristalización del hidroalumo-silicato de la solución.

Leiteizen (4) obtuvo una relación de tipo Arrhenius que caracteriza la cinética del proceso de disolución del $\mathrm{SiO}_{2}$ en las soluciones de aluminato de bauxitas con una cantidad relativamente elevadas de sílice:

$$
\ln \mathrm{K}=\frac{12.200}{22.65-\mathrm{T}}
$$


TABLA No.1.

ANALISIS GRANULOMETRICO DE LA PRUEBA DE BAUXITA

\begin{tabular}{|c|c|c|c|c|c|c|}
\hline \multicolumn{2}{|c|}{$\begin{array}{l}\text { Fracción } \\
\text { Granulométrica, mm }\end{array}$} & \multicolumn{3}{|c|}{ Contenido, \% } & \multicolumn{2}{|c|}{ Raz6n AL 203/si02 } \\
\hline \multirow[b]{2}{*}{$10-5$} & \multirow{2}{*}{$\frac{\mathrm{AL}_{2} \mathrm{O}_{3}}{42.45}$} & \multirow{2}{*}{$\frac{\mathrm{SiO}_{2}}{6.40}$} & \multirow{2}{*}{$\frac{\mathrm{Fe}_{2} \mathrm{O}_{3}}{25.20}$} & \multirow{2}{*}{$\frac{\mathrm{TIO} 2}{1.85}$} & \multicolumn{2}{|c|}{$\mathrm{PC}\left(100^{\circ} \mathrm{C}\right)$} \\
\hline & & & & & 23.55 & 6.60 \\
\hline $5-1$ & 43.65 & 7.25 & 22.40 & 1.80 & 24.60 & 6.00 \\
\hline 1. $0-0.25$ & 46.50 & 12.90 & 13.20 & 1.44 & 25.60 & 3.60 \\
\hline $0.25-0.10$ & 39.75 & 13.20 & 23.10 & 2.60 & 21.25 & 3.00 \\
\hline $0.10-0.03$ & 34.70 & 14.15 & 29.80 & 3.90 & 17.30 & 2.45 \\
\hline $0.03-0.005$ & 45.25 & 9.80 & 16.00 & 1.95 & 26.40 & 4.60 \\
\hline $0.005-0.002$ & 48.25 & 7.90 & 13.80 & 1.98 & 28.30 & 5.20 \\
\hline $0.002-0$ & 47.35 & 8.30 & 14.40 & 2.05 & 28.04 & 5.70 \\
\hline $10-0$ & 45.58 & 9.48 & 16.41 & 1.95 & 26.48 & 4.80 \\
\hline $10-0.03$ & 41.67 & 11.57 & 23.45 & 2.16 & 22.77 & 3.60 \\
\hline $0.03-0$ & 46.22 & 9.14 & 15.27 & 1.92 & 27.09 & 5.00 \\
\hline
\end{tabular}

en donde " $K$ " es la constante de equilibrio y " $T$ " la temperatura absoluta en grados Kelvin.

Cuando el contenido de $\mathrm{SiO}_{2}$ se acerca a la situación metaestable, la ecuación para condiciones estables de Leiteizen no tiene validez. En la realidad, conjuntamente con la disolución de $\mathrm{SiO} 2$ tiene lugar también su precipitación bajo la forma de hidroalumo-silicato. De esta manera la concentración de sílice en solución en los primeros momentos se eleva hasta un valor cercano al de la concentración de saturación y por lo tanto, la cinética de disolución del $\mathrm{SiO} 2$ queda sujeta a otras leyes diferentes para las que se obtuvo la ecuación (6).

Leiteizen estableció también la ecuación cinética (7) para la desilización con un agente de precipitación. Para esto supuso a desilización soluciones con $200 \mathrm{~g} / \mathrm{L} \mathrm{Na} a_{2} \mathrm{O} \quad \mathrm{K}=3.5$ y 1.2 a $5 \mathrm{~g} / \mathrm{L}$ $\mathrm{SiO}_{2}$. La desilización se llevó a cabo a $95^{\circ} \mathrm{C}$ en presencia del agente de precipitación (se usó como agente de precipitación el barro obtenido después de la fase de solubilización de la bauxita (barro rojo) que contiene cerca de $75 \%$ hidroalumo-silicato).

La ecuación obtenida por Leiteizen que caracteriza el proceso de desilización en el período inicial es la siguiente:

$$
\frac{\mathrm{dx}}{\overline{\mathrm{d} t}}=\mathrm{k}\left(\mathrm{X}-\mathrm{X}_{\mathrm{t}}\right)^{\mathrm{n}}
$$

Con esta ecuación se puede calcular la concentración de $\mathrm{SiO}_{2}$ en la fase líquida de la pulpa (X) como también la forma en que transcurre la cristalización del alumo-silicato referido a la velocidad de transformación del silice.

\section{Investigaciones Experimentales de Laboratorio}

El objetivo de las experiencias de laboratorio fue el de establecer la influencia de unos factores tecnológicos sobre la desilización de las soluciones de aluminato, con miras a establecer las condiciones óptimas de desilización de la pulpa bruta obtenida en tratamiento de las bauxitas con un contenido elevado de sílice por medio del proceso Bayer.

\subsection{Características de la materia prima utilizada}

En las tablas que se muestran a continuación se presentan los resultados del análisis granulométrico y las composiciones química y mineralogica de la bauxita utilizada. 
TABLA No. 2

COMPOSICION QUIMICA DE LA BAUXITA USADA

\begin{tabular}{|l|c|}
\hline Substancia \% & (en peso \\
\hline $\mathrm{Al}_{2} \mathrm{O}_{3}$ & 46,55 \\
$\mathrm{SiO}_{2}$ & 8,35 \\
$\mathrm{Fe}_{2} \mathrm{O}_{3}$ & 5,95 \\
$\mathrm{TiO}_{2}$ & 2,00 \\
Pérdidas por & 25,90 \\
calcinación & \\
Razón de & 5,57 \\
silice $\mathrm{Al}_{2} \mathrm{O}_{3} / \mathrm{SiO}_{2}$ & \\
\hline
\end{tabular}

TABLA No. 3

COMPOSICION MINERALOGICA DE LA PRUEBA DE BAUXITA

\begin{tabular}{|c|c|c|}
\hline Mineral & Fórmula & $\%$ \\
\hline $\begin{array}{l}\text { Hidrargilita } \\
\text { Diaspor } \\
\text { Caolinita } \\
\text { Cuarzo } \\
\text { Cristobalita } \\
\text { Goethita } \\
\text { Magnetita } \\
\text { Martita } \\
\text { Rutilo, } \\
\text { leucoxeno, } \\
\text { ilmenita }\end{array}$ & $\begin{array}{l}\mathrm{Al}(\mathrm{OH})_{3} \\
\mathrm{AlOOH} \\
\mathrm{Al}_{4} / \mathrm{Si}_{4} \mathrm{O}_{10} /(\mathrm{OH})_{8} \\
\mathrm{SiO}_{2} \\
\mathrm{FeOOH} \\
\mathrm{FeO}_{3} \mathrm{O}_{4} \\
\mathrm{Fe}_{3} \mathrm{O}_{4} \\
\mathrm{Fe}_{2} \mathrm{O}_{3} \\
\\
\mathrm{TiO}_{2}, \mathrm{TiO}_{2}+\mathrm{Fe}_{2} \mathrm{O}_{3}, \mathrm{~F}\end{array}$ & $\begin{array}{l}\sim 65 \\
\sim 2 \\
7 \sim 8 \\
\sim 5 \\
<1 \\
13 \sim 15 \\
\sim 2 \\
1 \sim 2\end{array}$ \\
\hline
\end{tabular}

\subsection{Condiciones Experimentales}

La pulpa bruta se obtuvo por el mezclado de la bauxita con la solución de recirculación (o solución de $\mathrm{NaOH}$ sintética) y óxido de calcio. En la práctica se introduce $\mathrm{CaO}$ en cantidad de $5 \%$ con respecto al peso de la bauxita. La composición de la pulpa bruta utilizada es el siguiente: $421 \mathrm{ml}$.; $\mathrm{H}_{2} \mathrm{O}, 167,74$ g. $\mathrm{NaOH}, 260$ g. bauxita $10.53 \mathrm{~g} \mathrm{CaO}$. En el cálculo de la cantidad de la solución de recirculación se tuvo en cuenta la obtención de una pulpa después de la fase de solubilización con un modo cáustico de

$$
\mathrm{aK}=1,7 \text {. }
$$

Temperatura: $95-105^{\circ} \mathrm{C}$. Tiempo $4-10$ horas.
Agitación: la velocidad de rotación del agitador fue cerca de $300 \mathrm{rev} / \mathrm{min}$.

\subsection{Instalación de Laboratorio y Modo de \\ Trabajo}

Las experiencias de laboratorio fueron realizadas con la ayuda de una instalación que comprendía en: un termostato, un agitador mecánico y un vaso tipo erlenmayer. La prueba de pulpa bruta se introdujo en un vaso de vidrio el cual se coloca en el termostato. La pulpa estudiada se agitó permanentemente y se recogieron pruebas para análisis en diferentes péríodos de tiempo. En el primer período ( 1.5 horas) el intervalo de tiempo de recolección de pruebas fue más pequeño. En estas condiciones se pudo estudiar la influencia de la temperatura, del tiempo de agitación y de unos agregados de desilización $(\mathrm{CaO})$, sobre el contenido de $\mathrm{SiO}_{2}$ de la solución. En el momento de las experiencias a la temperatura menor de $100^{\circ} \mathrm{C}$ se usó un termostato con agua y para la temperatura mayor de $100^{\circ} \mathrm{C}$ se usó un termostato con glicerina. La velocidad de rotación del agitador fue de cerca $300 \mathrm{rev} / \mathrm{min}$. El agua evaporada de la pulpa en el momento de las experiencias de desilización se completó por adiciones de agua destilada.

\section{Resultados Experimentales y sus Interpretaciones}

Con el objeto del establecimiento de condiciones optimas de desilización las experiencias efectuadas se realizaron en el dominio de temperaturas $90-105^{\circ} \mathrm{C}$. Los resultados obtenidos después de las experiencias de desilización a 95 y $105^{\circ} \mathrm{C}$, usando agregados de $\mathrm{CaO}$ se dan en las Tablas No. 4 y No. 5 . y en las Figuras No.1 y No. 2.

Del análisis de los resultados obtenidos resulta que con la agitación de la pulpa bruta, el contenido de $\mathrm{SiO}_{2}$ en solución crece hasta un valor máximo después del cual comienza a disminuir. Este fenómeno se explica de la forma siguiente: en la primera etapa, debido al contacto entre la solución de recirculación y la bauxita, tiene lugar el paso en solución del sílice hallado bajo la forma de mineral fácilmente soluble; súlice amorfo, caolinita, etc.. En la etapa siguiente del proceso de desilización como resultado de la interacción química entre el sílice disuelto en la primera fase y el aluminato de sodio, se forman alumo-silicatos insolubles que separándose llevan a la disminución 


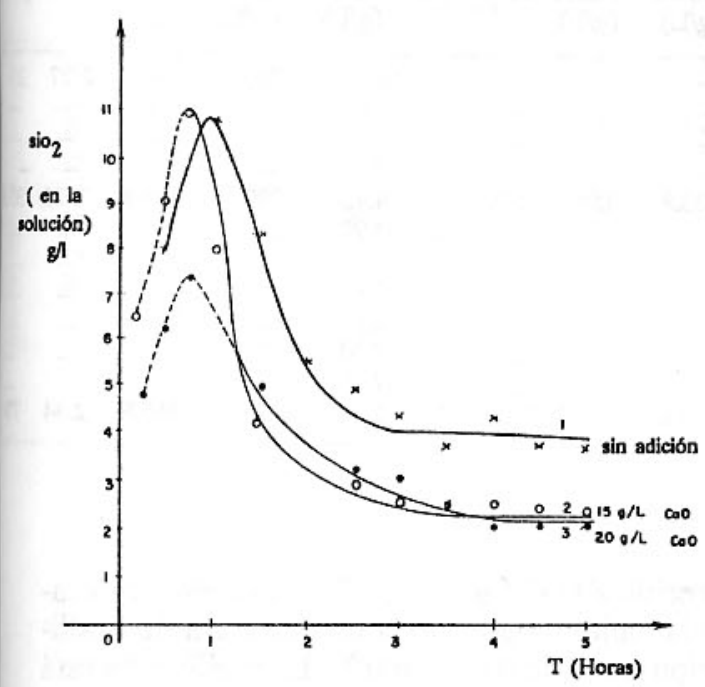

FIGURA No. 1. Comportamiento del bioxido de silicio en el proceso de desilización $\left(95^{\circ} \mathrm{C}\right)$.

del contenido de $\mathrm{SiO}_{2}$. Esto confirma los datos de la literatura de especialización según los cuales en el período inicial del contacto entre el sílice y la solución de aluminato, la velocidad de solubilización del sillice sobrepasa la velocidad de formación de los hidroalumo-silicatos poco solubles llegándose a una concentración máxima de $\mathrm{SiO}_{2}$ metaestable. $\mathrm{La}$ cantidad del $\mathrm{SiO}_{2}$, en equilibrio en esta fase está en función del límite de solubilidad del hidroalumo-silicato.

La cantidad máxima de sulice que pasa a la solución depende de la temperatura de trabajo, de la velocidad de agitación, de la naturaleza y concentración del agregado $(\mathrm{CaO})$. Del mismo modo el logro de este máximo, más rápido o más tardío, está en función de los mismos factores. A la temperatura de $95^{\circ} \mathrm{C}$ (tab. 4) la solubilidad máxima del sílice es de cerca 9.65 a $11,05 \mathrm{~g} / \mathrm{L}$ se logra después de 30-60 min. de agitación. De la tabla No.5 resulta que a la temperatura de $105^{\circ} \mathrm{C}$. La solubilidad máxima de $\mathrm{SiO}_{2}$ se logró rápido (en los primeros $30 \mathrm{~min}$. de agitación), y el valor de este máximo es mayor. Prácticamente, por determinaciones en intervalos de $30 \mathrm{~min}$., este máximo no se pudo establecer. Sin embargo, se puede afirmar que el máximo formado en la curva de desilización de la pulpa bruta en función del tiempo, es más pequeño y más aplanado conforme es más baja la temperatura. Después de lograr el máximo de solubilidad, el contenido de $\mathrm{SiO}_{2}$ disminuye rápidamente hasta un cierto límite donde continua casi constante.

En la desilización sin agregado (curva 1 Figura No. 1 y No. 2), el contenido de $\mathrm{SiO}_{2}$, despúes 5 horas de agitación, es cerca de $4 \mathrm{~g} / \mathrm{L}$ a la temperatura de $95^{\circ} \mathrm{C}$. Este fenómeno se explica por el hecho que a temperaturas más bajas se forman compuestos zeolíticos con solubilidad mayor, mientras que a temperaturas elevadas (sobre $100^{\circ} \mathrm{C}$ ) se forman sodalitos

La presencia de $15 \mathrm{~g} / \mathrm{L}^{3}$ y respectivamente 20 $\mathrm{g} / \mathrm{L}^{3} \mathrm{CaO}$ (curvas 2 y 3 Figura No. 1 y No. 2) disminuyen el contenido de $\mathrm{SiO}_{2}$ (en el caso de la desilizacion a $95^{\circ} \mathrm{C}$ de $4 \mathrm{~g} / \mathrm{L}$ en ausencia de $\mathrm{CaO}$, hasta un valor dentro del ámbito de 2 a $2.5 \mathrm{~g} / \mathrm{L}$ ). Este hecho se explica debido a la presencia de $\mathrm{CaO}_{3}$ que desde la primera etapa de la desilización, forma en una proporción elevada una serie de compuestos insolubles con base de $\mathrm{CaO}, \mathrm{SiO}_{2}$, $\mathrm{Al}_{2} \mathrm{O}_{3}$, lo que contribuye a la eliminación de una gran cantidad de $\mathrm{SiO}_{2}$ incluso en esta etapa.

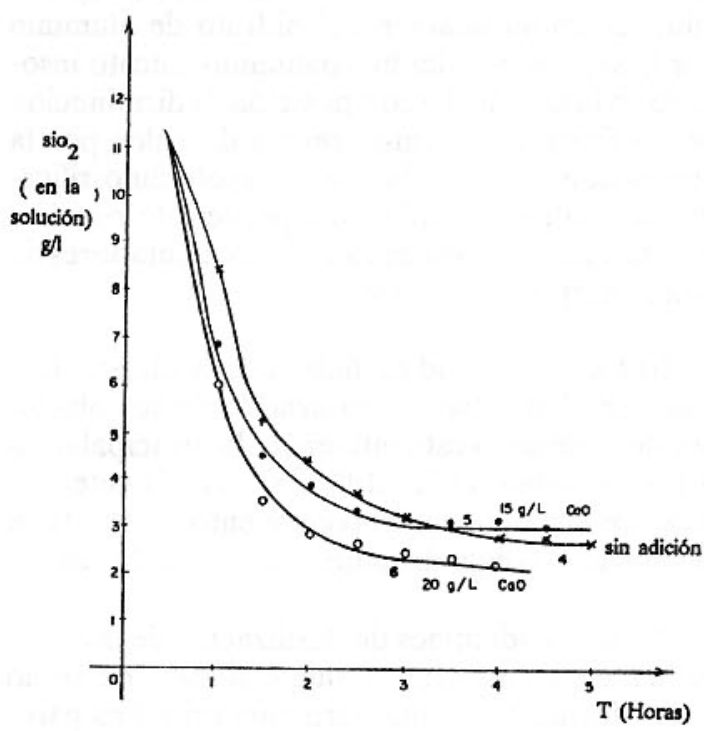

FIGURA No. 2. Comportamiento del bioxido de silicio en el proceso de desilizacion $\left(105^{\circ} \mathrm{C}\right)$. 


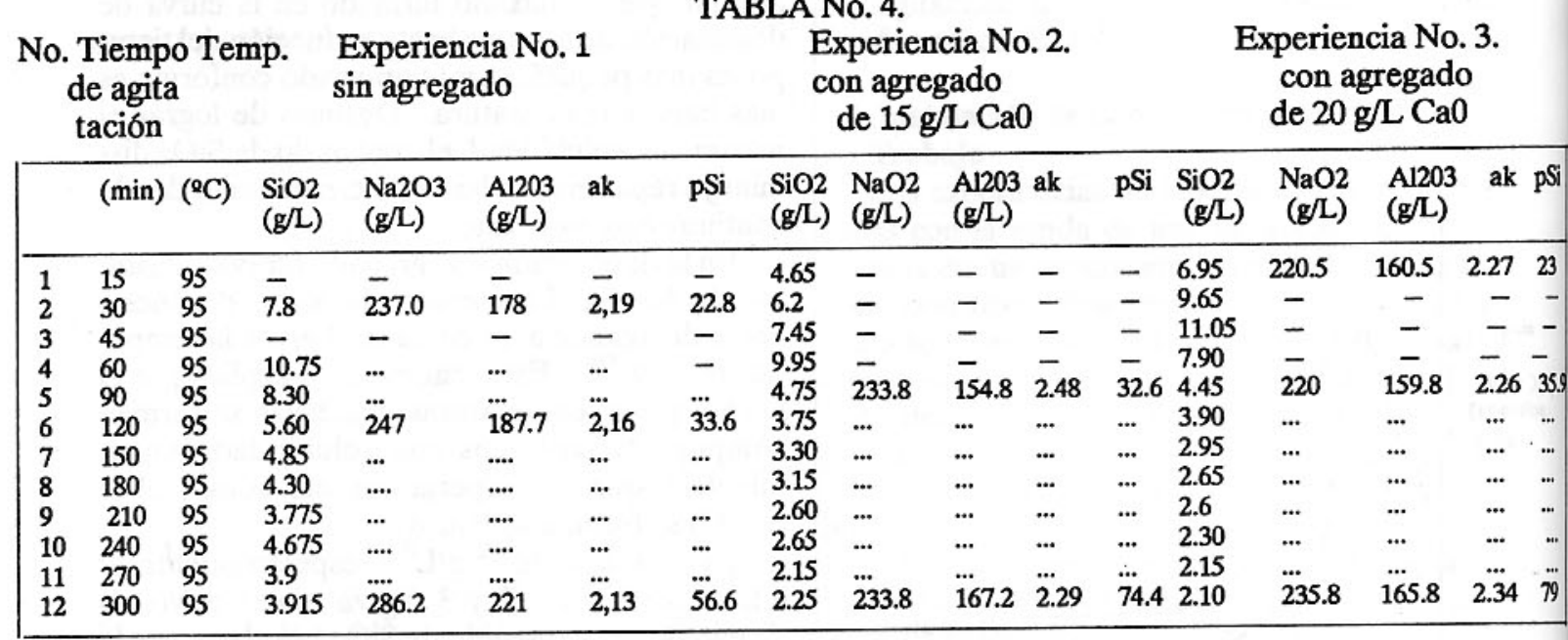

\section{Conclusiones}

a) En el proceso Bayer, los minerales de silicio presentes en la bauxita reaccionan en forma aproximadamente cuantitativa con las soluciones alcalinas de alumintao. El contenido de $\mathrm{SiO}_{2}$ en la fase líquida de la pulpa cruda sobrepasa varias a veces al límite de solubilidad del $\mathrm{SiO}_{2}$. El sílice en exceso precipita bajo la forma de hidroaluminosilicatos de sodio y calcio. Estas combinaciones, en especial el hidroalumo-silicato, llevan una serie de inconvenientes como: pérdidas de soda y alúmina, la impurificación del hidrato de aluminio por la separación del hidroalumino-silicato insoluble en la fase de descomposición, la disminución de coeficiente de transferencia de calor por la deposición de partículas (del hidroalumino-silicato principalmente) sobre la superficie de los tubos de intercambiadores de calor (precalentadores de pulpa, evaporadores, etc.)

b) La solubilidad de hidroalumo-silicato, formado en el proceso de desilización de las soluciones de aluminato esta influenciado principalmente por el contenido de $\mathrm{Al}_{2} \mathrm{O}_{3}$, es decir el contenido de silice aumenta con el crecimiento de $\mathrm{Al}_{2} \mathrm{O}_{3}$ en la solución (con la disminución, del modo caústico).

En las condiciones de desilización de las soluciones de aluminato, el alumo-silicato de sodio que se forma, tiene una estructura cristalina parecida a la de las zeolitas, y se puede transformar ulteriormente en una estructura más densa de tipo parecido a la del sodalito. Este proceso se desarrolla mejor, conforme la temperatura de desilización crece de $80^{\circ} \mathrm{C}$ a $100^{\circ} \mathrm{C}$. La explicación está en el solubilidad relativamente elevada de los compuestos zeolíticos en comparación con la solubilidad relativamente reducida de los sodalitos.

c) El crecimiento de la temperatura influencia favorablemente el proceso de desilización. El contenido de $\mathrm{SiO}_{2}$, en la fase líquida de la pulpa bruta, después de la primera etapa de desilización, es menor en el caso de las temperaturas elevadas (sobre $100^{\circ} \mathrm{C}$ ). $\mathrm{Al}$ mismo tiempo, valores pequeños del contenido de $\mathrm{SiO}_{2}$ se obtiene en tiempo de agitación menor en el caso de las temperaturas mayores, sin embargo, en base a las experiencias efectuadas a $95^{\circ} \mathrm{C}$ y $105^{\circ} \mathrm{C}$, resultó que es más conveniente que el proceso de desilización sea conducido a $95^{\circ} \mathrm{C}$ debido al hecho que a las temperaturas mayores de $100^{\circ} \mathrm{C}$ se intensifica el proceso de evaporación de la pulpa.

d) El tiempo de duración de la agitación influencia favorablemente el proceso de desilización. Es decir conforme la duración de la agitación es mayor, el contenido de $\mathrm{SiO}_{2}$ en la solución es menor. Con base a las experiencias efectuadas resultó que la duración óptima de agitación esta en función de la temperatura. Para la temperatura de $95^{\circ} \mathrm{C}$ la duración óptima de agitación es de 4 a 5 horas. 
No. Tiempo Temp. Experiencia No. 4 de agita tación
TABLA No. 5.

Experiencia No.5. con agregado de $15 \mathrm{~g} / \mathrm{L} \mathrm{Ca} 0$
Experiencia No. 6. con agregado de $20 \mathrm{~g} / \mathrm{L} \mathrm{Ca} 0$

\begin{tabular}{|c|c|c|c|c|c|c|c|c|c|c|c|c|c|c|c|c|}
\hline & $(\min )$ & $\left({ }^{\circ} \mathrm{C}\right)$ & $\begin{array}{l}\mathrm{SiO} 2 \\
(\mathrm{~g} / \mathrm{L})\end{array}$ & $\begin{array}{l}\mathrm{Na} 2 \mathrm{O} 3 \\
(\mathrm{~g} / \mathrm{L})\end{array}$ & $\begin{array}{l}\text { Al203 } \\
(\mathrm{g} / \mathrm{L})\end{array}$ & ak & $\mathrm{pSi}$ & $\begin{array}{l}\mathrm{SiO2} \\
(\mathrm{g} / \mathrm{L})\end{array}$ & $\begin{array}{l}\mathrm{NaO2} \\
(\mathrm{g} / \mathrm{L})\end{array}$ & $\begin{array}{l}\mathrm{Al} 203 \\
(\mathrm{~g} / \mathrm{L})\end{array}$ & ak & $\mathrm{pSi}$ & $\begin{array}{c}\mathrm{SiO} 2 \\
(\mathrm{~g} / \mathrm{L})\end{array}$ & $\begin{array}{c}\mathrm{NaO} 2 \\
(\mathrm{~g} / \mathrm{L})\end{array}$ & $\begin{array}{l}\mathrm{Al} 203 \\
(\mathrm{~g} / \mathrm{L})\end{array}$ & ak pS \\
\hline 1 & 30 & 105 & 11.05 & 462.11 & 127.5 & 5.96 & 11.53 & 11.25 & 440.52 & 113,47 & 7638 & 10.1 & 10.9 & 231.6 & 183.5 & $207 \quad 23$ \\
\hline 2 & 60 & 105 & 8.3 & 481.29 & 130.556 & 6.06 & 15.7 & 6.45 & - & $\ldots$ & - & - & 12.8 & $\ldots$ & - & - \\
\hline 3 & 90 & 105 & 5.2 & 501.4 & 126.2 & 6.53 & 24.2 & 4.45 & 559.3. & 142.8 & 6.34 & 32.1 & 3.35 & $\ldots$ & $\ldots$ & $\ldots$ \\
\hline 4 & 120 & 105 & 4.4 & 497.28 & 121.3 & 6.74 & 27.5 & 4.40 & 523.71 & 126.2 & 6.82 & 28.4 & 2.85 & 236.4 & 174.93 & $2.22-$ \\
\hline 5 & 150 & 105 & 4.15 & 479.74 & 120.5 & 4.58 & 29 & 3.37 & 522.3 & 126.8 & 6.81 & 37.8 & 2.57 & $\ldots$ & $\ldots$ & $\ldots \quad \ldots$ \\
\hline 6 & 180 & 105 & 3.95 & 489.09 & 122,7 & 6.51 & 31 & 3.20 & 518.92 & 115 & 7.43 & 35.9 & 2.47 & $\ldots$ & $\ldots$ & $\ldots$ \\
\hline 7 & 210 & 105 & 3.20 & $\ldots$. & $\ldots$ & $\ldots$ & $\ldots$ & 3.10 & 520.51 & 115 & 7.44 & 37.1 & 2.25 & & & $\begin{array}{ll}\ldots & . .\end{array}$ \\
\hline 8 & 240 & 105 & 2.80 & .... & $\ldots .$. & $\ldots$. & $\ldots$ & 3.15 & 532.52 & 110.8 & 7.93 & 34.8 & 2.22 & 251.11 & 190.5 & 2.16. 85.8 \\
\hline 9 & 270 & 105 & 2.70 & $\ldots$ & $\ldots$ & $\ldots$ & $\ldots$ & 2.90 & $\ldots$ & $\ldots$ & $\ldots$ & $\cdots$ & $\ldots$ & $\ldots$ & $\ldots$ & $\quad \cdots \quad$ \\
\hline 10 & 300 & 105 & 2.57 & 498.2. & 125.2 & 6.5 & 48.8 & 285 & 522.14 & 210.45 & .8 .23 & 36.7 & $\ldots$ & $\ldots$ & $\ldots$ & $\ldots$ \\
\hline
\end{tabular}

e) Las experiencias efectuadas con diferentes cantidades de agregado $(\mathrm{CaO})$ pusieron en evidencia una intensificación acentuada del proceso de desilización al aumentarse la cantidad de óxido de calcio.

\section{BIBLIOGRAFIA}

1. Perry, K.E. Modifications to Bayer Digestion (ALCOA). ICSOBA 1969. P/3.

Brever, R.C. - Barsotti, L.R. - Kelly, A.C. Behaviour of silica in sodium - aluminate solutions - Extractive Methallurgy of Aluminium. Vol. I. p. 133.
2. Maliseu, H.P. - Tuetnia Metallurghia 6, 1961. pg. 65.

3. Lileev, I.S., Pesin, A.M., Trudi Giph. 1936, p. 25.

4. Leiteizen, M. G.,Leigehei Metalli.nr.10, 1936, p. 54.

5. Ni, L.P., Rerehrest, G.P. Jumal PridadnoiHimii 35, 1962, p. 944

6. Snirnor, M.N. Jomal PridadnoiHimii,37, 1964, p. 16.

7. Pevsner,I.Z, Lainer, A.I.s.a Izv. VuzTvetnaia Metallurghia, nr.4, 1968, p.35.

8. Siziacov, V.M., Smirnou, M.G. Tvetnie Metalli, nr. 6, 1969, p.56.

9. Pevsner, I.Z., Lainer, AI, TvetnieMetalle, nr.9, 1970, p.26.

10. Petehrest, Solenko - Jornal PhihladnoiHimii, 1, 1964, pg. 22. 